\title{
Medicamentos y prolongación del intervalo QT
}

\section{Introducción}

La actividad eléctrica del corazón se divide en dos fases: despolarización y repolarización.

La despolarización resulta del flujo neto de cargas positivas (paso a través de la membrana de sodio y calcio) hacia el interior de la célula, lo que estimula la contracción cardiaca y se representa en el trazado electrocardiográfico por el intervalo QRS.

La repolarización es el resultado de la salida de cargas positivas (potasio) a través de la membrana hacia el exterior celular, con lo cual la célula miocárdica regresa al estado de reposo donde pueda ser nuevamente estimulada por el segmento ST y la onda T. (Ver figura \#1)

Figura \#1 Representación esquemática de un trazado electrocardiográfico normal

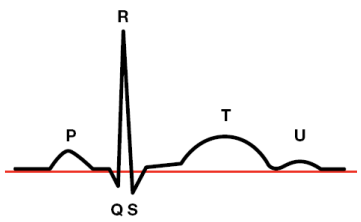

La prolongación del intervalo QT es una alteración causada por alargamiento de la fase de repolarización del potencial de acción ventricular. Se caracteriza por la prolongación del intervalo QT, con o sin alteraciones de la onda $T$, en el electrocardiograma, junto con una dispersión aumentada de la repolarización ventricular que se asocia a la predisposición de arritmias ventriculares malignas tipo Torsade de Pointes (TdP) y en algunas ocasiones fibrilación ventricu- lar que puede conducir a una muerte súbita cardiaca. (Ver figura \#2).

Figura \#2 Electrocardiograma normal y electrocardiograma de un paciente con elevación del intervalo QT

Figura 2a. Tira de ritmo de un paciente con electrocardiograma normal.

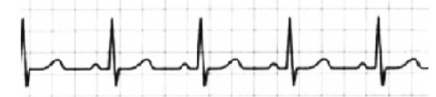

Figura 2b. Tira de ritmo de un paciente con SQTL.

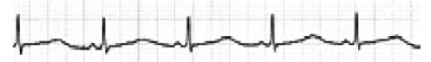

La prolongación del intervalo QT puede tener un origen congénito debido a una mutación en canales iónicos o un origen adquirido, en general por la toma de algunos medicamentos.

En los últimos años uno de los motivos más frecuentes de retirada del mercado de medicamentos o de restricciones de uso ha sido la prolongación del intervalo QT. A pesar de que su prevalencia es baja, la gravedad de la aparición de un episodio por esta causa puede tener consecuencias mortales.

En la actualidad, la prolongación del intervalo QT con o sin efecto proarrítmico asociado es junto con la hepatotoxicidad, la causa más común de retirada de fármacos comercializados.

El objetivo de este boletín es realizar una revisión de los fármacos que podrían prolongar el intervalo QT.

\section{Centro de Información de Medicamentos \\ Servicio de Farmacia HSJD}

Volumen 5, Número 7 Julio 2015

- "La prolongación del intervalo QT con o sin efecto proarrítmico asociado, es junto con la hepatotoxicidad la causa más común de retirada de fármacos comercializados"

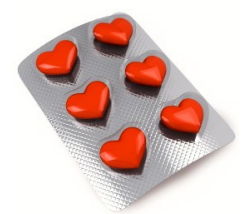

\section{Contenido:}

\begin{tabular}{|l|l|}
\hline $\begin{array}{l}\text { Fármacos que poten- } \\
\text { cialmente aumentan el } \\
\text { intervalo QT }\end{array}$ & 2 \\
\hline $\begin{array}{l}\text { Interacciones farmaco- } \\
\text { lógicas que aumentan el } \\
\text { intervalo QT }\end{array}$ & 4 \\
\hline $\begin{array}{l}\text { Alertas de } \\
\text { Farmacovigilancia }\end{array}$ & 5 \\
\hline
\end{tabular}




\section{Medicamentos y prolongación del intervalo QT}

En el mercado farmacéutico existen más de 100 fármacos con capacidad de prolongar el intervalo QT.

Esta reacción adversa es inducida por distintos compuestos químicos utilizados en el tratamiento de una amplia variedad de patologías, lo cual implica un alto riesgo de asociación de estos fármacos.

Una de las complicaciones de esta reacción adversa es la de provocar TdP. La probabilidad de desarrollar TdP se asocia a factores como comorbilidad, edad avanzada, alteración hepática o renal, enfermedad cardiaca de base o alteraciones electrolíticas. En bradicardia, el intervalo QT tiende a ser más largo, mientras que es más corto en presencia de taquicardia.

También está sujeto a variaciones diurnas, así se incrementa durante el sueño, siendo mayor a primeras horas de la mañana y en el periodo postprandial.

Actualmente, las distintas agencias reguladoras exigen la identificación del posible riesgo de prolongación del intervalo QT en fases de preautorización y posautorización de medicamentos.
La Agencia Europea del Medicamento (EMA) en 2005 elaboró un documento donde se recogen las recomendaciones para la evaluación de la prolongación del intervalo QT y el potencial proarrítmico para medicamentos no antiarrítimicos. A partir de estas directrices la FDA publicó ese mismo año una guía dirigida a la industria, donde se recogían las pautas para la evaluación clínica de prolongación del intervalo QT antes de comercializar un medicamento en los Estados Unidos.

\section{Principales grupos de fármacos que potencialmente afectan el intervalo QT}

El mecanismo por el que determinados medicamentos prolongan el intervalo QT se debe generalmente a que bloquean los canales de potasio cardiacos.

La incidencia de TdP producida por medicamentos no está establecida, aunque se presupone que es muy baja.

Entre los medicamentos más conocidos que prolongan el intervalo QT están los antiarrítmicos. Sin embargo, se ha observado que hay más fármacos que presentan este efecto secundario como algunos antihistamínicos, antibióticos, antivirales, antimicóticos, antieméticos, neurolépticos, antidepresivos, entre otros.

La prolongación del intervalo QT podría darse a dosis superiores a las recomendadas o a las dosis recomendadas si se administran concomitantemente con medicamentos que inhiben el metabolismo enzimáticos del citocromo P-450.
En los últimos años por esta causa se han retirado medicamentos como: astemizol y terfenadina ya que su empleo implicaba mayor riesgo que beneficio terapéutico.

A continuación una descripción de los grupos terapéuticos que potencialmente afectan al intervalo QT:

Antiarrítmicos: es el único grupo de medicamentos sobre el que se tienen más datos disponibles acerca de su asociación con la prolongación del intervalo QT y TdP.

Antihistamínicos: especialmente los de segunda generación (caso de la terfenadina), aparecen cuando se utilizan a dosis superiores a las recomendadas o a dosis habituales cuando se administran concomitantemente con inhibidores del citocromo P-450 o en pacientes con insuficiencia hepática.

Los antihistamínicos de segunda generación como loratadina, ceterizina que son de más reciente comercialización no se han relacionado con prolonga-

\section{ción del QT ni TdP.}

Antibióticos: no es muy frecuente y los casos que se conocen han sido debidos al uso de macrólidos y fluroquinolonas. Dentro de los macrólidos se ha relacionado a la eritromicina y a la claritromicina con arritmias ventriculares. La azitromicina se ha relacionado con casos de TdP, principalmente en pacientes con enfermedad cardiovascular de base.

Las fluorquinolonas constituyen el segundo grupo de antibióticos que afectan el intervalo QT, de todo el grupo la ciprofloxacina parece ser la más segura. La información disponible revela que los casos de TdP asociados a fluorquinolonas ocurrió cuando se administraron asociadas a otros fármacos que prolongan el intervalo QT.

El Ketoconazol, itraconazol, fluconzaol y voriconazol se han relacionado con casos de aumento del intervalo QT y aparición de TdP 


\section{Continuación, Principales grupos de fármacos que potencialmente afectan el intervalo QT}

Ketoconazol e itraconazol prolongan el intervalo QT por bloqueo de los canales de potasio, pero la mayoría de los casos notificados se han debido a la coadministración con otros fármacos que incrementan el intervalo QT que se metabolizan por la misma vía enzimática.

Antimaláricos: especialmente quinina y quinidina ya que pueden aumentar el intervalo QT a dosis habituales. Además su cardiotoxicidad aumenta en enfermos renales.

Antivirales- inhibidores de proteasa: incrementan el intervalo QT ya que se asocian a un bloqueo de los canales HERG.

Antidepresivos y antipsicóticos: su incidencia no se conoce con exactitud, el uso de antidepresivos tricíclicos como la amitriptilina y la imipramina se han asociado a la prolongación del intervalo QT y aparición de TdP. EI citalopram solo se ha asociado con nuevos casos de TdP en pacientes sobredosificados.

En cuánto a los fármacos antipsicóticos relacionados con la prolongación del intervalo QT, se han notificado muertes súbitas tanto por su uso a dosis normales como por sobredosis.

El Arizona Center for Education and Research on Therapeutics, el cual es un centro de referencia mundial en este ámbito, a través de su página web ofrece listas actualizadas de medicamentos comercializados en Estados Unidos y en el resto del mundo que potencialmente prolongan el intervalo QT y aumentan el riesgo de sufrir TdP. En esta página se clasifican los fármacos en función del grado de atribución de la relación causal entre el medicamento y el acontecimiento.

De esta forma se clasifican tres grupos :

Definida: existe evidencia de que el uso de estos fármacos puede provocar una prolongación del intervalo QT y riesgo de TdP.

Posible: existe evidencia de que pueden prolongar el intervalo QT, pero no hay evidencia suficiente de riesgo de TdP.

Condicionada: la evidencia sostiene que podrían prolongar el intervalo QT solo bajo determinadas condiciones (dosis excesiva, interacciones medicamentosas, etc).

En la siguiente tabla se proporciona una lista de los fármacos de uso frecuente en el país que pueden prolongar el intervalo QT.

\section{Tabla \#1 Fármacos que pueden prolongar el intervalo QT}

\begin{tabular}{|c|c|c|c|}
\hline Categoría & \multicolumn{3}{|c|}{ Fármacos } \\
\hline Definida & $\begin{array}{l}\text { Clozapina } \\
\text { Haloperidol } \\
\text { Citalopram } \\
\text { Azitromicina } \\
\text { Claritromicina } \\
\text { Eritromicina } \\
\text { Arsénico, trióxido } \\
\text { Domperidona } \\
\text { Tizanidina }\end{array}$ & $\begin{array}{l}\text { Amiodarona } \\
\text { Procainamida } \\
\text { Quinidina } \\
\text { Alfuzosina } \\
\text { Prometazina } \\
\text { Tamoxifeno } \\
\text { Difenhidramina } \\
\text { Metadona }\end{array}$ & $\begin{array}{l}\text { Ondasetrón } \\
\text { Vemurafenib } \\
\text { Famotidina } \\
\text { Fingolimod } \\
\text { Granisetrón } \\
\text { Lapatinib } \\
\text { Nilotinib } \\
\text { Tacrolimus }\end{array}$ \\
\hline Posible & $\begin{array}{l}\text { Clorpromazina } \\
\text { Litio } \\
\text { Olanzapina } \\
\text { Quetiapina }\end{array}$ & $\begin{array}{l}\text { Risperidona } \\
\text { Mirtazapina } \\
\text { Venlafaxina } \\
\text { Norfloxacino }\end{array}$ & $\begin{array}{l}\text { Levofloxacino } \\
\text { Ofloxacino } \\
\text { Saquinavir } \\
\text { Voriconazol }\end{array}$ \\
\hline Condicionada & $\begin{array}{l}\text { Amitriptilina } \\
\text { Fluoxetina } \\
\text { Ciprofloxacino } \\
\text { Ketoconazol }\end{array}$ & $\begin{array}{l}\text { Imipramina } \\
\text { Paroxetina } \\
\text { Fluxonazol } \\
\text { Ritonavir }\end{array}$ & $\begin{array}{l}\text { Sertralina } \\
\text { Amantadina } \\
\text { Itraconazol } \\
\text { Metilfenidato }\end{array}$ \\
\hline
\end{tabular}


En la práctica clínica las interacciones farmacológicas habitualmente se dan por desconocimiento del prescriptor sobre las mismas o porque, a pesar del riesgo potencial, el beneficio es mayor o no existe alternativa terapéutica para determinada patología.

El riesgo se incrementa por sinergia cuando se usan a la vez varios medicamentos que aumentan el intervalo QT.

El riesgo es mayo en pacientes hospitalizados, generalmente porque su situación clínica es más compleja y porque requiere nuevos tratamientos añadidos .

En la tabla \#2 se detallan algunos ejemplos de interacciones farmacológicas más comunes y en la tabla \#3 las isoenzimas del citocromo P-450 involucradas en el metabolismo oxidativo de medicamentos involucrados en el metabolismo oxidativo de medicamentos que prolongan la repolarización ventricular.

\section{Tabla \#2 Interacciones farmacocinéticas de fármacos que prolongan el intervalo QT}

\begin{tabular}{|c|c|l|}
\hline Grupo de fármacos & $\begin{array}{c}\text { Fármaco que aumenta } \\
\text { el intervalo QT }\end{array}$ & Fármaco que interactúa \\
\hline Antiarrítmicos & Procainamida & $\begin{array}{l}\text { Eritromicina } \\
\text { Amiodarona } \\
\text { Cimetidina } \\
\text { Trimetroprim }\end{array}$ \\
\hline Antipsicóticos & Haloperidol & $\begin{array}{l}\text { Fluoxetina } \\
\text { Venlafaxina } \\
\text { Fluconazol } \\
\text { Itraconazol } \\
\text { Ketoconazol }\end{array}$ \\
\hline Antidepresivos & Amitriptilina & $\begin{array}{l}\text { Cimetidina } \\
\text { Fluconazol } \\
\text { Fluoxetina } \\
\text { Ritonavir }\end{array}$ \\
\hline Antibióticos & Eritromicina & $\begin{array}{l}\text { Venlafaxina } \\
\text { Ritonavir }\end{array}$ \\
\hline
\end{tabular}

Tabla \#3 Isoenzimas del citocromo P-450 involucradas en el metabolismo oxidativo de medicamentos que prolongan la repolarización ventricular

\begin{tabular}{|c|c|c|c|c|}
\hline CYP1A 2 & CYP2C9 & CYP2C19 & CYP2D6 & CYP3A4 \\
\hline $\begin{array}{l}\text { Amitriptilina } \\
\text { Clomipramina } \\
\text { Imipramina }\end{array}$ & $\begin{array}{c}\text { Amitriptilina } \\
\text { Clomipramina } \\
\text { Fluoxetina } \\
\text { Imipramina } \\
\text { Voriconazol }\end{array}$ & $\begin{array}{l}\text { Amitriptilina } \\
\text { Clomipramina } \\
\text { Imipramina } \\
\text { Voriconazol }\end{array}$ & $\begin{array}{l}\text { Amitriptilina } \\
\text { Clorpromazina } \\
\text { Fluoxetina } \\
\text { Haloperidol } \\
\text { Imipramina } \\
\text { Metadona } \\
\text { Ondasetrón } \\
\text { Paroxetina } \\
\text { Quetiapina } \\
\text { Risperidona }\end{array}$ & $\begin{array}{c}\text { Amitriptilina } \\
\text { Amiodarona } \\
\text { Claritromicina } \\
\text { Eritromicina } \\
\text { Haloperidol } \\
\text { Imipramina } \\
\text { Itraconazol } \\
\text { Ketoconazol } \\
\text { Metadona } \\
\text { Ondasetrón } \\
\text { Quetiapina } \\
\text { Quinidina } \\
\text { Tacrolimus }\end{array}$ \\
\hline
\end{tabular}

Tomado de Revista Argentina de Cardiología, Vol 12 N6, Noviembre-Diciembre 2004 


\section{Conclusiones y recomendaciones}

La muerte súbita cardiaca constituye un importante problema de salud pública. La mayoría de los casos derivan de arritmias ventriculares agudas y se deben a enfermedad coronaria o bien a consecuencia de la prolongación del intervalo QT y un importante factor de riesgo para estas alteraciones es el uso de fármacos que prolongan el intervalo QT.

Además, muchos de estos fármacos que prolongan el intervalo QT son no cardiovasculares y no se tiene conciencia de este riesgo. Por tanto, instituciones como el American College of Cardiology, la Amercian Heart Association y la European Society of Cardiology han establecido una serie de recomendaciones para la prevención de TdP inducida por fármacos, las cuales se enuncian a continuación:

1. Conocer los medicamentos que pueden prolongar el intervalo QT

2. Conocer factores de riesgo de TdP inducido por fármacos (edad avanzada, sexo femenino, enfermedad celiaca, alteraciones electrolíticas, enfermedad renal o hepática, bradicardia, antecedentes familiares o predisposición genética)

3. Tratamiento concomitante con otros medicamentos que incrementan el intervalo QT o posibilidad de interacciones farmacocinéticas o farmacodinámicas.

4. Antes de iniciar un tratamiento con un medicamento que incremente el intervalo QT valorar de forma individualizada el riesgo/beneficio y las posibles alternativas terapéuticas disponibles.

5. Determinar el valor del QT antes de iniciar tratamientos de este tipo.

6. Informar al paciente sobre el riesgo del uso de ciertos medicamentos e interacciones y proporcionarle documentación escrita adecuada.

\section{Bibliografía}

1. Boletín de Información Terapéutica de Navarra. Medicamentos y Prolongación del Intervalo QT, Volumen Número 21, Enero- Marzo 2013, disponible en http://www.navarra.es/NR/rdonlyres/C6A41120-7788-42D3-8374 -38A8F2C8D727/257184/Bit_v21n1.pdf, consultado el 10 de agosto 2015

2. Villamañán, E, et al. Prolongación del intervalo QT inducido por fármacos. Medicina Clínica 2015; 144(6): 269274

3. Hocht, C, et al. Intervalo QT prolongado inducido por fármacos desde el punto de vista de un farmacólogo. Revista Argentina de Cardiología. Vol 72 N6/Noviembre- Diciembre 2004

\section{Alertas de Farmacovigilancia}

A continuación un resumen de las alertas de Farmacovigilancia más importantes emitidas por las agencias reguladoras internacionales.

1. Riesgos potenciales de usar medicamentos para la tos y gripe que contengan codeína:

La FDA está investigando los posibles riesgos de utilizar medicamentos que contengan codeína para el tratamiento de tos y gripe en niños menores de 18 años de edad debido al potencial de sufrir efectos secundarios graves, incluyendo respiración lenta o dificultosa. La FDA está evaluando toda la información disponible y también consultará con expertos externos convocando un comité asesor para discutir estos problemas de salud. La FDA informará las conclusiones finales cuando la revisión haya concluido. En Abril de 2015, la Agencia Europea de Medicamentos (EMA) anunció que la codeína no debe ser utilizada para el tratamiento de tos y gripe en niños menores de 12 años y que no se recomienda codeína en niños y adolescentes entre los 12 y 18 años de edad que tengan dificultades respiratorias, incluyendo aquellos con asma y otros problemas respiratorios crónicos. La FDA continuará evaluando este problema de salud y considerará las recomendaciones de la EMA.

http://www.fda.gov/Drugs/DrugSafety/ucm453850.htm 


\section{Continuación de Alertas de Farmacovigilancia}

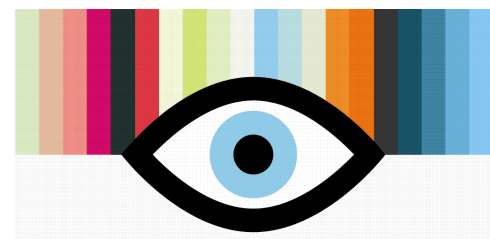

FARMACOVIGILANCIA

\section{AINES}

La FDA está reforzando la advertencia existente en la etiqueta de AINES sin aspirina ya que aumentan la probabilidad de ataque cardíaco o derrame cerebral.

Basados en la revisión integral de nueva información de seguridad se está solicitando actualizaciones a las etiquetas de información farmacológica en todos los medicamentos AINES prescritos.

Los pacientes que toman AINES deben buscar atención médica de inmediato si experimentan síntomas tales como dolor de pecho, falta de aire o problemas al respirar, debilidad en una parte - lado del cuerpo o dificultad súbita para hablar.

http://www.fda.gov/Drugs/ DrugSafety/ucm454962.htm, 7/7/15

\section{Hidroxicina}

La Agencia Europea del Medicamento (EMA) actualizó la advertencia de contraindicar la administración de este medicamento a pacientes con intervalo QT aumentado o con factores de riesgo de prolongación de este intervalo, además disminuyó la dosis máxima en adultos a $100 \mathrm{mg} /$ día e inclusive en adultos mayores se prefiere la dosis de $50 \mathrm{mg} /$ día.

Estas recomendaciones fueron avaladas por el Comité de Evaluación de Riesgos de la Agencia Europea del Medicamento

Drug Safety Update, MHRA Volumen 8, issue 9: April 2015 (www.gov.uk/ mhra)

4. Vacuna virus del papiloma humano La EMA esta iniciando una revisión del perfil de seguridad de la vacuna del virus del papiloma humano. Se calcula que esta vacuna ha sido utilizada por al menos 72 millones de personas alrededor del mundo y propiamente esta revisión hará énfasis en dos condiciones Síndrome de dolor regional complejo y Síndrome de taquicardia postural ortostática (condición en la que se acelera el ritmo cardiaco después de sentarse o ponerse de pie, causando mareo y fatiga, así como dolor de cabeza, dolor en el pecho y debilidad).

A partir de los resultados de esta revisión se espera determinar si es necesario hacer cambios en el etiquetado del medicamento.

Ema.europa.eu, Human Medicines Highlights July 2015

\section{Hospital San Juan de Dios Servicio de Farmacia Centro de Información de Medicamentos}

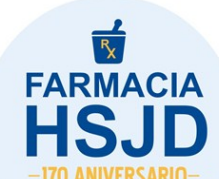

Elaborado por: Dra. Cristina Fernández Barrantes, Farmacéutica

Consultas:

- $\quad$ Teléfono: 2547-8324

- $\quad$ Correo electrónico: cimf_hsjd@ccss.sa.cr

Puede encontrar números anteriores del boletín en:

Revista Clínica- Hospital San Juan de Dios/ Escuela de Medicina UCR

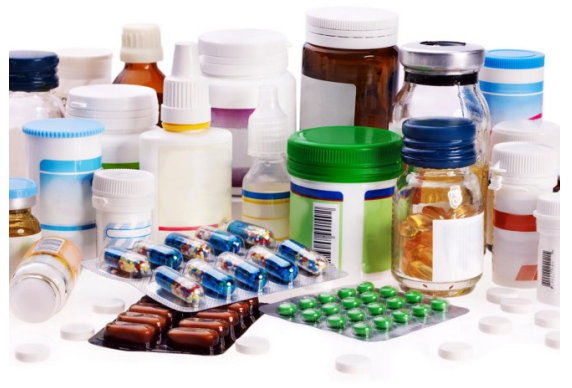

